Trends in Horticulture (2018) Volume 1

doi: $10.24294 /$ th.v 1 ii 2.889

\title{
Stem transcriptome of cold stressed Eucalyptus globulus and E. urograndis
}

\section{Pedro Araújo $^{1}$, Felipe Thadeu Tolentino ${ }^{2}$, Adilson Pereira Domingues Junior ${ }^{3}$, Flávia Camila Schimpl ${ }^{4}$, Daniela Feltrim², Vanessa Tofanello ${ }^{2}$, Nathália Volpi ${ }^{2}$, Jullyana Cristina Magalhães Silva Moura Sobczak $^{5}$ and Paulo Mazzafera ${ }^{2,3}$}

${ }^{1}$ Department of Genetic, Evolution, Microbiology and Immunology, Institute of Biology, State University of Campinas, São Paulo, Brasil

${ }^{2}$ Department of Plant Biology, Institute of Biology, State University of Campinas, São Paulo, Brasil

${ }^{3}$ Laboratório de Produção Vegetal, Escola Superior de Agricultura "Luiz de Queiroz", University of São Paulo, Piracicaba, São Paulo, Brasil

${ }^{4}$ Faculty of Agricultural Science, Federal University of Amazon, Manaus, AM, Brasil

${ }^{5}$ Department of Agronomy, Universidade da Integração Internacional da Lusofonia Afro-Brasileira, Redenção, Ceará, Brasil

\begin{abstract}
Eucalyptus is an important source of cellulose and a widely cultivated plant. Biotechnology tools can save time spent in breeding and transcriptomic approaches generate a gene profile that allows the identification of candidates involved in processes of interest. RNA-seq is a commonly used technology for transcript analysis and it provides an overview of regulatory pathways. Here, we selected two contrasting Eucalyptus species for cold acclimatization and focused in responsive genes under cold condition aiming woody properties - lignin and cellulose. The number of differentially expressed genes identified in stem sections were 3.300 in Eucalyptus globulus and 1370 in Eucalyptus urograndis. We listed genes with expression higher than 10 times including NAC, MYB and DUF family members. The GO analysis indicates increased oxidative process for E. urograndis. This data can provide information for more detailed analyses for breeding, especially in perennial plants.
\end{abstract}

Keywords: Eucalyptus; RNA-seq; Cold Stress; Cell Wall

\section{Introduction}

Eucalyptus is one of the most cultivated tropical trees for cellulose pulp production and it has been suggested for use as an alternative for biofuel production ${ }^{[1]}$. Species in this genus are widespread in the world as a crop due to their large range of adaptability on diverse climates. Eucalyptus species are grown all over the Australian continent and nearby islands, including Tasmania, in its coldest parts. Eucalyptus is mostly cultivated in tropical regions, although there is great interest to expand to subtropical and temperate climate ${ }^{[2]}$. Some species, such as E. globulus, E. viminalis, E. dunnii and E. gunnii can grow in low temperatures but so far, it has been shown that only E. gunnii seems to resist freezing temperature ${ }^{[3]}$. E. globulus wood has the highest $\mathrm{S} / \mathrm{G}$ (syringyl/guaiacyl) ratio, a characteristic that confers low recalcitrance for lignin removal and, consequently high cellulose yield. Under high temperature the S/G ratio of E. globulus decreases, which is probably related with the worst growth performance of these trees compared with other species adapted to tropical temperature ${ }^{[4]}$. Other species exploited commercially such as E. saligna, E. urophylla, E. grandis, E cloezina and C. citriodora grow better in warmer places ${ }^{[5,6]}$. E. grandis, E. urophylla and the hybrid E. grandis $\mathrm{x}$ E. urophylla are the most planted Eucalypts in Brazil, showing rapid growth and good fiber quality ${ }^{[7]}$.

The genetic mechanism behind cold tolerance is still unclear despite many genes that have been characterized Several genes coordinate the response to cold, by altering lipid composition and activating $\mathrm{Ca}^{2+}$ channels, which is me-

Copyright (C) 2018 Pedro Araújo et al.

doi: $10.24294 /$ th.v1i2.889

EnPress Publisher LLC.This work is licensed under the Creative Commons Attribution-NonCommercial 4.0 International License (CC BY-NC 4.0).

http://creativecommons.org/licenses/by/4.0/ 
diated by reactive oxygen species and abscisic acid. Cold-regulated genes may range from $4 \%$ to $20 \%$ of the whole genome in Arabidopsis ${ }^{[8]}$.

RNA-seq is a useful tool to study transcriptome profiling, providing a wide overview of different functional elements in the genome ${ }^{[9]}$. The quantification of these elements can provide specific information on the developmental stage and physiological conditions, thus enabling comparisons between samples under different treatments or conditions $^{[9]}$.

We sequenced a transcript profile of stems from seedlings of E. globulus and E. urograndis grown under low temperature. We detected differential expression of several genes focused in cold stress tolerance and economically important traits for Eucalyptus breeding. Our study provides an overview of two contrasting species of Eucalyptus and enumerates several differential expression genes.

\section{Methods}

\subsection{Plant material, RNA extraction and sequencing}

Plants of E. globulus and E. urograndis were obtained from seeds collected from clonal gardens (Caiçara Sementes - http://www.sementescaicara.com) and cultivated in a greenhouse for 180 days, without control of humidity and temperature. Then the plants were transferred to growth chambers set to $4{ }^{\circ} \mathrm{C}$ or $25^{\circ} \mathrm{C}$, at $300 \mu \mathrm{mol} \mathrm{m} \mathrm{m}^{-2} \mathrm{~s}^{-1}$ and an $8 \mathrm{~h}$ photoperiod for 30 days. Stems were collected starting at $5 \mathrm{~cm}$ from the soil and a pool for each species were used for RNA extraction.

\subsection{RNA extraction}

Trizol (Invitrogen) was used for RNA extraction following the manufacturer protocol. The RNA quantity and quality were verified using Nanodrop 2000 (Thermo scientific) and agarose gel.

\subsection{RNA sequencing}

Performed by Fasteris (DNA Sequencing Service - https://www.fasteris.com/dna/) using SN365 - HiSeq2000, number of cycling $1 \times 100+7$ (index). Sequencing was carried out on four samples: (i) E. globulus control (11.394.167 reads); (ii) E. globulus cold stress (19.973.014 reads); (iii) E. urograndis control (16.151.851 reads) and (iv) E. urograndis cold stress (15.779.681). Quality control was performed and had an average of $79.9 \%$ of Q30 score per base.

\subsection{RNA-seq analysis}

The software GeneSpring (Agilent) was used to analyze our dataset from Fasteris. Initially, the reads were aligned against Eucalyptus grandis database from Phytozome (www.phytozome.net) - 8X mapped E. grandis BRASUZ1 genome assembly and annotation were carried out with Avadis (version 1.1 within GeneSpring software, Agilent). We also excluded ribosomal and low quality reads. All genes aligned/identified (known-genes and predicted ones) were analyzed separately to evaluate the expression pattern in each treatment and only reads found in all four libraries were selected to calculate the fold change. We also performed scatter-plotting graphics to identify relationships in gene expression (fold change) between treatments on both species. The same was done for the predicted new genes (not annotated).

All genes identified using E. grandis genome as reference were functionally annotated using Blast2Go and Arabidopsis thaliana annotation. The expression levels of each gene were determined using the FPKM (fragments per kilobase of exon per million fragments) value. Data from the cold treatments were normalized using the data from the corresponding control plants of each Eucalyptus species and the normalized data was used to compare differentially expressed genes between species. A differentially expressed gene was defined using two cut-offs 1 and $10\left(\log _{2}\right)$.

\section{Results}

\section{1 mRNA distribution of two contrasting species of Eucalyptus}

To evaluate the transcriptional response of E. globulus and E. urograndis to cold stress we performed a broad analysis of the sequenced transcripts. Figure 1A shows that 13.380 genes are shared between the two species and 3.306 and 1.376 genes are uniquely expressed in E. urograndis and E. globulus, respectively. Figure 1B shows control and cold treatments data for both species. It is evident that E. urograndis regulates more unique genes than E. globulus. In the former 2007 and 1299 annotated genes were down- and upregulated, respectively, while 741 and 625 were found in 


\section{E. globulus.}

GeneSpring software aligns annotated reads and suggests "predicted new genes", which were not annotated, using E. grandis genome as reference. The software identified as "new" 253 upregulated and 333 downregulated genes (Figure 1B). In contrast, 246 genes were upregulated in E. globulus but downregulated in E. urograndis and conversely 381 genes were downregulated in E. globulus and upregulated in E. urograndis.

A
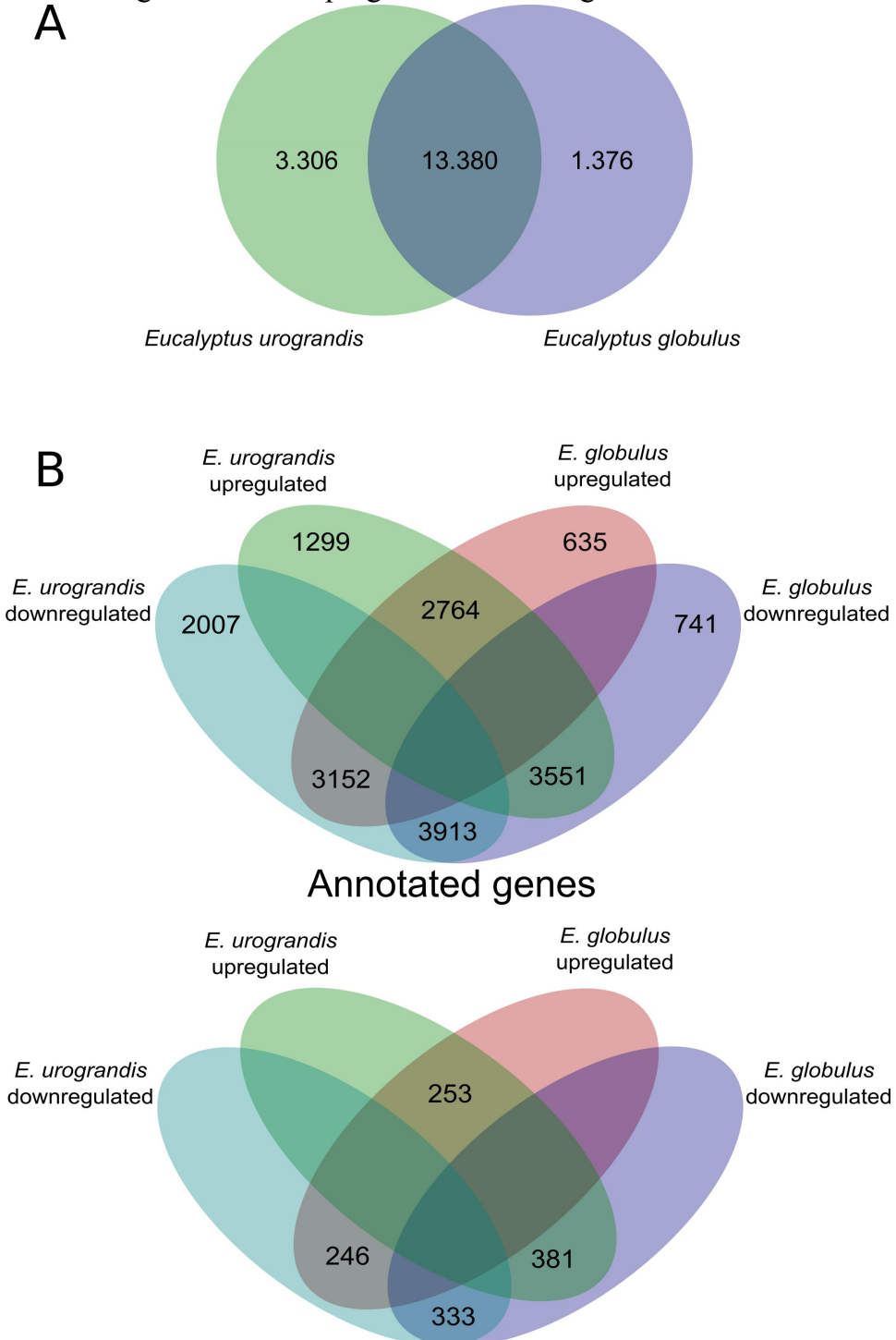

Predicted new genes

Figure 1; Overview of all annotated genes expressed under cold treatment for E. globulus (purple) and E. urograndis (green). They shared 13.380 genes for cold stress, being 3.306 genes differentially expressed for E. urograndis and 1.376 genes for E. globulus (A). Differentially expressed annotated genes and predicted new new genes by GeneSpring software (B).

A spatial distribution from Figure 1 is shown in Figure 2. We explored the data to identify the most differentially expressed genes in Eucalyptus species. We used a cut-off of 10 to check the most differentially expressed genes found in both species. A cloud of genes (spots) tends to be aggregated in the middle close to line 0 for both species. E. globulus contributes for a more sparse distribution with higher levels of difference for up and down regulation. Two extreme sides were selected and a gene list was summarized on Tables 1 and 2. There is a brief description and function of each selected gene and Arabidopsis homolog. Several uncharacterized genes were present and some were without homologs (Eucgr.E03184). Interesting and well annotated homologs for these genes can be found: members of DUF genes: Eucgr.I02070, Eucgr.I02359, Eucgr.H03259 and Eucgr.L02157; NAC: Eucgr.C02105 and Eucgr.A02070 and MYB: Eucgr.A00996. 




Eucalyptus globulus

Figure 2; Distribution of gene expression profile between E. globulus and E. urograndis. We selected the outmost genes representing both species for detailed identification. Table 1 shows a list of genes upregulated for E. globulus cold and downregulated for E. urograndis cold and Table 2 shows a list of genes downregulated for E. globulus cold and upregulated for E. urograndis cold.

\begin{tabular}{|c|c|c|c|}
\hline Gene ID & $\begin{array}{c}\text { Arabidopsis } \\
\text { homolog* }\end{array}$ & Description & Function* \\
\hline Eucgr.F02784 & AT1G75050 & $\begin{array}{l}\text { Pathogenesis-related } \\
\text { superfamily protein }\end{array}$ & Uncharacterized function and localized on endomembrane \\
\hline Eucgr.E00767 & AT1G01490 & $\begin{array}{l}\text { Heavy metal transport/detoxification } \\
\text { superfamily protein }\end{array}$ & Metal ion transport \\
\hline Eucgr.E01683 & AT1G60320 & $\begin{array}{lcr}\text { Disease } & \text { resistance } & \text { protein } \\
\text { (TIR-NBS-LRR class) family } & \end{array}$ & $\begin{array}{l}\text { Transmembrane receptor activity involved in signal } \\
\text { transduction }\end{array}$ \\
\hline Eucgr.J00988 & AT4G27190 & $\begin{array}{l}\text { NB-ARC domain-containing disease } \\
\text { resistance protein }\end{array}$ & $\begin{array}{l}\text { NB-ARC domain-containing disease resistance protein, } \\
\text { involved in apoptosis, defense response }\end{array}$ \\
\hline Eucgr.H01219 & AT3G44550 & fatty acid reductase 4 & $\begin{array}{l}\text { Member of gene family encoding alcohol-forming fatty } \\
\text { acyl-CoA reductases (FARs) }\end{array}$ \\
\hline Eucgr.B00127 & AT1G76650 & calmodulin like 37 & Calcium ion binding on plasma membrane \\
\hline Eucgr.F02812 & AT2G01300 & - & Uncharacterized \\
\hline Eucgr.L01926 & - & - & - \\
\hline Eucgr.B01326 & AT5G26680 & $5 \backslash^{\prime}-3 \backslash^{\prime}$ exonuclease family protein & DNA binding and nuclease activity \\
\hline Eucgr.C01418 & AT2G42160 & zinc finger domain-containing protein & $\begin{array}{l}\text { Protein form a heteromeric complex required for the } \\
\text { development }\end{array}$ \\
\hline Eucgr.G01737 & AT2G21250 & $\begin{array}{l}\text { NAD }(P) \text {-linked } \quad \text { oxidoreductase } \\
\text { superfamily protein }\end{array}$ & Oxidoreductase activity in response to cadmium ion \\
\hline Eucgr.H03343 & AT1G19640 & S-adenosyl-L-methionine-dependent & Catalyzes the formation of methyljasmonate from \\
\hline
\end{tabular}




\begin{tabular}{llll}
\hline Eucgr.B00621 & AT1G18610 & $\begin{array}{l}\text { Galactose oxidase/kelch } \\
\text { superfamily protein }\end{array}$ & $\begin{array}{l}\text { repeat } \\
\text { Uncharacterized }\end{array}$ \\
\hline Eucgr.H01117 & AT1G09220 & $\begin{array}{l}\text { Pentatricopeptide } \\
\text { superfamily protein }\end{array}$ & $\begin{array}{l}\text { repeat } \\
\text { (PPR) Uncharacterized function and localized on cytosolic } \\
\text { ribosome }\end{array}$ \\
\hline Eucgr.L02664 & - & beta-ketoacyl reductase 1 & $\begin{array}{l}\text { catalyzes the first reduction during very long chain fatty } \\
\text { acids, >18 carbon elongation }\end{array}$ \\
\hline Eucgr.K00822 & AT3G21820 & $\begin{array}{l}\text { histone-lysine } \\
\text { ATXR2 }\end{array}$ & N-methyltransferase \\
Zinc ion binding and unknown function
\end{tabular}

Table 1. Spatial gene distribution between E. globulus and E. urograndis. Genes upregulated in E. globulus and independently for E. urograndis (Figure 2). Cut-off fold change: 10. We also indicate the Arabidopsis homolog, description and function using TAIR database.

\begin{tabular}{llll}
\hline Gene ID & $\begin{array}{l}\text { Arabidopsis } \\
\text { homolog* }\end{array}$ & Description & Function* \\
\hline Eucgr.G00235 & AT4G24280 & chloroplast heat shock protein 70-2 & $\begin{array}{l}\text { Involved in protein import into chloroplasts during early } \\
\text { developmental stages }\end{array}$ \\
\hline \multirow{2}{*}{ Eucgr.F03098 } & AT1G73040 & $\begin{array}{l}\text { Mannose-binding lectin superfamily } \\
\text { protein }\end{array}$ & Mannose-binding lectin superfamily protein \\
\hline
\end{tabular}

\section{Eucgr.C02554 AT5G23960 terpene synthase 21}

Encodes a sesquiterpene synthase involved in generating all of the group. A sesquiterpenes found in the Arabidopsis floral volatile blend.

\begin{tabular}{|c|c|c|c|}
\hline Eucgr.A01557 & AT5G12100 & $\begin{array}{l}\text { pentatricopeptide } \\
\text { repeat-containing protein }\end{array}$ & pentatricopeptide $(\mathrm{PPR})$ repeat-containing protein \\
\hline Eucgr.C03056 & AT2G26150 & heat shock transcription factor $\mathrm{A} 2$ & $\begin{array}{l}\text { Heat Stress Transcription Factor (Hsf) family. Involved in } \\
\text { response to misfolded protein accumulation in the cytosol. }\end{array}$ \\
\hline Eucgr.H02642 & AT1G05560 & $\begin{array}{l}\text { indole-3-acetate beta-D-glucosyltransfe } \\
\text { rase }\end{array}$ & $\begin{array}{l}\text { A UDP-glucose transferase localized in the phragmoplast. } \\
\text { It has been co-purified with the callose synthase complex } \\
\text { and may transfer UDP-glucose from sucrose synthase to } \\
\text { the callose synthase and thus help form a substrate } \\
\text { channel for the synthesis of callose at the forming cell } \\
\text { plate. Induced by salicylic acid. }\end{array}$ \\
\hline Eucgr.E00383 & AT4G19050 & $\begin{array}{l}\text { NB-ARC domain-containing disease } \\
\text { resistance protein }\end{array}$ & ATP binding. Involved in defense response and apoptosis \\
\hline Eucgr.D02256 & AT2G24190 & $\begin{array}{l}\text { NAD(P)-binding } \\
\text { superfamily protein }\end{array}$ & $\begin{array}{l}\text { Encodes an aldehyde reductase that catalyzes the } \\
\text { reduction of the aldehyde carbonyl groups on saturated }\end{array}$ \\
\hline
\end{tabular}


and alpha,beta-unsaturated aldehydes with more than 5 carbons in vitro. In addition, this enzyme can reduce methylglyoxal in vitro. It is believed that this enzyme is believed to be localizeds to the cytosol like such as the closely related protein encoded by AT3G61220

\begin{tabular}{|c|c|c|c|}
\hline Eucgr.H03259 & AT1G56230 & $\begin{array}{l}\text { Protein of unknown function } \\
\text { (DUF1399) }\end{array}$ & Unknown function and expressed in plasma membrane \\
\hline Eucgr.F00207 & AT5G67090 & $\begin{array}{l}\text { Subtilisin-like serine endopeptidase } \\
\text { family protein }\end{array}$ & $\begin{array}{l}\text { Subtilisin-like serine endopeptidase family protein. } \\
\text { Involved in proteolysis, negative regulation of catalytic } \\
\text { activity on endomembrane system }\end{array}$ \\
\hline Eucgr.A02555 & AT5G06400 & $\begin{array}{lll}\text { Pentatricopeptide repeat } & (\mathrm{PPR}) \\
\text { superfamily protein } & & \end{array}$ & Pentatricopeptide repeat (PPR) superfamily protein \\
\hline Eucgr.H01277 & AT2G18360 & $\begin{array}{l}\text { alpha/beta-Hydrolases superfamily } \\
\text { protein }\end{array}$ & $\begin{array}{l}\text { alpha/beta-Hydrolases superfamily protein; hydrolase } \\
\text { activity in endomembrane system; expressed in shoot } \\
\text { apex, hypocotyl, root, leaf }\end{array}$ \\
\hline Eucgr.C03936 & AT2G19710 & $\begin{array}{l}\text { Regulator of Vps4 activity in the MVB } \\
\text { pathway protein }\end{array}$ & $\begin{array}{l}\text { Regulator of Vps4 activity in the MVB pathway protein. } \\
\text { Unknown function. }\end{array}$ \\
\hline Eucgr.F00173 & AT4G37360 & cytochrome P450 & member of CYP81D \\
\hline Eucgr.B03206 & AT1G14140 & $\begin{array}{l}\text { Mitochondrial substrate carrier family } \\
\text { protein }\end{array}$ & $\begin{array}{l}\text { Transmembrane transporter activity. Mitochondrial } \\
\text { substrate carrier family protein }\end{array}$ \\
\hline Eucgr.A00876 & AT3G18150 & RNI-like superfamily protein & RNI-like superfamily protein \\
\hline Eucgr.H01261 & AT4G36670 & Major facilitator superfamily protein & $\begin{array}{l}\text { Major facilitator superfamily protein; carbohydrate } \\
\text { transmembrane transporter activity, transmembrane } \\
\text { transport }\end{array}$ \\
\hline Eucgr.I00512 & AT1G22360 & UDP-glucosyl transferase $85 \mathrm{~A} 7$ & $\begin{array}{l}\text { UDP-glucosyl transferase 85A2 (UGT85A2); } \\
\text { UDP-glycosyltransferase activity, transferase activity, } \\
\text { transferring glycosyl groups, glucuronosyltransferase } \\
\text { activity }\end{array}$ \\
\hline Eucgr.B03070 & AT1G68740 & $\begin{array}{l}\text { EXS (ERD1/XPR1/SYG1) family } \\
\text { protein }\end{array}$ & $\begin{array}{l}\text { Involved in inorganic phosphate }(\mathrm{Pi}) \text { transport and } \\
\text { homeostasis }\end{array}$ \\
\hline Eucgr.E00581 & AT3G48990 & $\begin{array}{l}\text { AMP-dependent synthetase and ligase } \\
\text { family protein }\end{array}$ & $\begin{array}{l}\text { Encodes an oxalyl-CoA synthetase and is required for } \\
\text { oxalate degradation }\end{array}$ \\
\hline Eucgr.E03184 & - & $\begin{array}{l}\text { LRR and NB-ARC domains-containing } \\
\text { disease resistance protein }\end{array}$ & - \\
\hline Eucgr.F00195 & AT5G42830 & $\begin{array}{l}\text { HXXXD-type acyl-transferase family } \\
\text { protein }\end{array}$ & $\begin{array}{l}\text { HXXXD-type acyl-transferase family protein, transferase } \\
\text { activity, transferring acyl groups other than amino-acyl } \\
\text { groups }\end{array}$ \\
\hline Eucgr.L00154 & AT5G47635 & len Ole e 1 allergen and extensin & Pollen Ole e 1 allergen and extensin family protein; \\
\hline
\end{tabular}




\begin{tabular}{|c|c|c|c|}
\hline & & family protein & located in endomembrane system. \\
\hline Eucgr.C02105 & AT2G24430 & NAC domain containing protein 38 & NAC Transcription factor (ANAC039) \\
\hline Eucgr.H02515 & AT1G10700 & $\begin{array}{l}\text { phosphoribosyl pyrophosphate (PRPP) } \\
\text { synthase } 3\end{array}$ & $\begin{array}{l}\text { Encodes a P-independent phosphoribosyl pyrophosphate } \\
\text { (PRPP) synthase }\end{array}$ \\
\hline Eucgr.G02674 & AT1G79480 & $\begin{array}{l}\text { Carbohydrate-binding } \mathrm{X} 8 \text { domain } \\
\text { superfamily protein }\end{array}$ & $\begin{array}{l}\text { Carbohydrate-binding X8 domain superfamily protein; } \\
\text { located in endomembrane system }\end{array}$ \\
\hline Eucgr.I02302 & AT4G35790 & phospholipase D delta & $\begin{array}{l}\text { Encodes a protein with phospholipase D activity. Involved } \\
\text { in phospholipase metabolism. Mutants are affected in } \\
\text { hydrogen peroxide- mediated cell death. }\end{array}$ \\
\hline Eucgr.D00506 & AT3G49340 & senescence-associated gene 12 & $\begin{array}{l}\text { Cysteine proteinases superfamily protein involved in } \\
\text { proteolysis }\end{array}$ \\
\hline Eucgr.K01946 & AT4G20910 & $\begin{array}{lcc}\text { double-stranded } & \text { RNA } & \text { binding } \\
\text { protein-related } & / & \text { DsRBD } \\
\text { protein-related } & & \end{array}$ & $\begin{array}{l}\text { Encodes an enhancer of hual and hua2 that acts to specify } \\
\text { reproductive organ identities and to repress A gene } \\
\text { function. }\end{array}$ \\
\hline Eucgr.I02070 & AT2G21080 & - & Unknown function. Similar to DUF3537 \\
\hline Eucgr.A01691 & AT2G36970 & $\begin{array}{l}\text { UDP-Glycosyltransferase superfamily } \\
\text { protein }\end{array}$ & $\begin{array}{l}\text { transferase activity, transferring glycosyl groups localized } \\
\text { on endomembrane }\end{array}$ \\
\hline Eucgr.K02813 & AT5G40390 & Raffinose synthase family protein & $\begin{array}{l}\text { Encodes a protein which might be involved in the } \\
\text { formation of verbascose }\end{array}$ \\
\hline Eucgr.F02756 & AT1G17950 & myb domain protein 52 & R2R3-MYB transcription family \\
\hline Eucgr.C01774 & AT4G31980 & - & Unknown protein and function \\
\hline Eucgr.A00388 & AT5G16990 & $\begin{array}{l}\text { Zinc-binding dehydrogenase family } \\
\text { protein }\end{array}$ & Oxidative stress tolerance \\
\hline Eucgr.H00964 & AT1G75820 & $\begin{array}{l}\text { Leucine-rich receptor-like protein } \\
\text { kinase family protein }\end{array}$ & $\begin{array}{l}\text { Putative receptor kinase with an extracellular leucine-rich } \\
\text { domain. Controls shoot and floral meristem size, and } \\
\text { contributes to establish and maintain floral meristem } \\
\text { identity }\end{array}$ \\
\hline Eucgr.F03704 & AT3G14810 & $\begin{array}{l}\text { mechanosensitive channel of small } \\
\text { conductance-like } 6\end{array}$ & $\begin{array}{l}\text { mechanosensitive channel of small conductance-like } 5 \\
\text { (MSL5); INVOLVED IN: transmembrane transport }\end{array}$ \\
\hline Eucgr.D02334 & AT2G46240 & BCL-2-associated athanogene 6 & regulators of apoptosis \\
\hline Eucgr.D00319 & AT1G29930 & chlorophyll A/B binding protein 1 & $\begin{array}{l}\text { Subunit of light-harvesting complex II (LHCII), which } \\
\text { absorbs light and transfers energy to the photosynthetic } \\
\text { reaction center }\end{array}$ \\
\hline Eucgr.A00996 & AT4G01680 & myb domain protein 55 & Encodes a putative transcription factor (MYB55) \\
\hline Eucgr.H03111 & AT1G06620 & $\begin{array}{l}\text { 2-oxoglutarate } \quad(2 \mathrm{OG}) \quad \text { and } \\
\mathrm{Fe}(\mathrm{II}) \text {-dependent oxygenase protein }\end{array}$ & similar to a 2-oxoglutarate-dependent dioxygenase \\
\hline
\end{tabular}




\begin{tabular}{|c|c|c|c|}
\hline Eucgr.F03960 & AT2G23060 & $\begin{array}{l}\text { Acyl-CoA N-acyltransferases } \quad(\mathrm{NAT}) \\
\text { superfamily protein }\end{array}$ & Acyl-CoA N-acyltransferases (NAT) superfamily protein \\
\hline Eucgr.A00632 & AT2G38460 & iron regulated 2 & iron efflux transporter ferroportin \\
\hline Eucgr.B02572 & AT1G70140 & $\begin{array}{l}\text { Actin-binding } \mathrm{FH} 2 \text { (formin homology } \\
\text { 2) family protein }\end{array}$ & $\begin{array}{l}\text { Binds to F-actin barbed ends. Has severing actin filaments } \\
\text { activity. InvovledInvolved in growth and cytoskeleton }\end{array}$ \\
\hline Eucgr.E03270 & AT4G20970 & $\begin{array}{l}\text { basic helix-loop-helix } \quad(\mathrm{bHLH}) \\
\text { DNA-binding superfamily protein }\end{array}$ & $\begin{array}{l}\text { DNA binding - transcription factor activity. } \\
\text { InvovledInvolved in defense response to fungus, } \\
\text { regulation of transcription }\end{array}$ \\
\hline Eucgr.G01747 & AT4G08850 & $\begin{array}{l}\text { Leucine-rich repeat receptor-like } \\
\text { protein kinase family protein }\end{array}$ & $\begin{array}{l}\text { Leucine-rich repeat receptor-like protein kinase family } \\
\text { protein; kinase activity; involved in protein amino acid } \\
\text { phosphorylation on plasma membrane }\end{array}$ \\
\hline
\end{tabular}

\begin{tabular}{|c|c|c|c|}
\hline Eucgr.K03566 & AT1G09390 & $\begin{array}{l}\text { GDSL-like Lipase/Acylhydrolase } \\
\text { superfamily protein }\end{array}$ & $\begin{array}{l}\text { GDSL-like Lipase/Acylhydrolase superfamily protein; } \\
\text { involved in glycerol biosynthetic process, lipid metabolic } \\
\text { process. }\end{array}$ \\
\hline Eucgr.G00487 & AT2G23690 & - & petal differentiation and expansion stage \\
\hline Eucgr.F03623 & AT5G41040 & $\begin{array}{l}\text { HXXXD-type acyl-transferase family } \\
\text { protein }\end{array}$ & $\begin{array}{l}\text { Encodes a feruloyl-CoA transferase required for suberin } \\
\text { synthesis. It Hhas feruloyl-CoA-dependent feruloyl } \\
\text { transferase activity towards substrates with a primary } \\
\text { alcohol. }\end{array}$ \\
\hline
\end{tabular}

\begin{tabular}{llll}
\hline Eucgr.K01062 & AT1G03840 & $\begin{array}{l}\mathrm{C} 2 \mathrm{H} 2 \text { and } \mathrm{C} 2 \mathrm{HC} \text { zinc fingers } \\
\text { superfamily protein }\end{array}$ & $\begin{array}{l}\text { MGP is a nuclear-localized putative transcription factor } \\
\text { with three zinc finger domains. MGP can interact with } \\
\text { three proteins implicated in root patterning. }\end{array}$ \\
\hline Eucgr.I02359 & AT1G19835 & $\begin{array}{l}\text { Plant protein of unknown function } \\
\text { (DUF869) }\end{array}$ & \\
\hline
\end{tabular}

$\begin{array}{llll}\text { Eucgr.D00192 AT4G21120 amino acid transporter 1 } & \text { (CAT) subfamily of amino acid polyamine choline } \\ & \text { transporters. Mediates efficient uptake of Lys, Arg and } \\ & \text { Glu in a yeast system. }\end{array}$

Encodes a member of the cationic amino acid transporter

\begin{tabular}{lll}
\hline Eucgr.F04109 AT4G10250 & $\begin{array}{l}\text { HSP20-like chaperones superfamily } \\
\text { protein }\end{array}$ & \\
\hline
\end{tabular}

Eucgr.A01605 AT3G53150 UDP-glucosyl transferase 73D1

\begin{tabular}{lllll} 
& & on endomembrane & \\
\hline Eucgr.K00184 & AT4G25420 & gibberellin 20 oxidase 2 & $\begin{array}{l}\text { Encodes gibberellin 20-oxidase that is involved in the } \\
\text { later steps of the gibberellin biosynthetic pathway. } \\
\text { Regulated by a circadian clock. Weak expression response } \\
\text { to far red light. }\end{array}$ \\
\hline \multirow{2}{*}{ Eucgr.J01884 } & AT3G08510 & $\begin{array}{l}\text { Phosphoinositide-specific } \\
\text { phospholipase C family protein }\end{array}$ & $\begin{array}{l}\text { Phosphoinositide-specific phospholipase C (PI-PLC), } \\
\text { catalyzes hydrolysis }\end{array}$ & posphatidylinositol \\
\hline
\end{tabular}


4,5-bisphosphate into inositol 1,4,5-trisphosphate and diacylglycerol.

Encodes a protein disulfide isomerase-like (PDIL) protein, a member of a multigene family within the Eucgr.K01503 AT3G03860 APR-like 5 thioredoxin (TRX) superfamily. This protein also belongs to the adenosine 5'-phosphosulfate reductase-like (APRL) group.

\begin{tabular}{llll}
\hline Eucgr.L00141 & Ankyrin repeat family protein & - \\
\hline Eucgr.C02224 & - & - \\
\hline
\end{tabular}

Eucgr.A02746 ATCG00430 photosystem II reaction center protein G

Encodes a protein which was originally thought to be part of photosystem II but its wheat homolog was later shown to encode for subunit $\mathrm{K}$ of NADH dehydrogenase

\begin{tabular}{llll}
\hline Eucgr.K02809 & AT5G63090 & $\begin{array}{l}\text { Lateral organ boundaries } \\
\text { domain family protein }\end{array}$ & $\begin{array}{l}\text { (LOB) } \\
\text { Involved in lateral organ development }\end{array}$ \\
\hline Eucgr.E00650 & - & - & - \\
\hline
\end{tabular}

Eucgr.C03553 AT5G09380 RNA polymerase III RPC4

DNA-directed RNA polymerase activity. transcription from RNA polymerase III promoter

\begin{tabular}{|c|c|c|c|}
\hline Eucgr.L01480 & AT1G34420 & $\begin{array}{l}\text { leucine-rich repeat transmembrane } \\
\text { protein kinase family protein }\end{array}$ & $\begin{array}{l}\text { Leucine-rich repeat transmembrane protein kinase family } \\
\text { protein; function in protein kinase activity, ATP binding } \\
\text { and involved in protein amino acid phosphorylation }\end{array}$ \\
\hline Eucgr.F01690 & AT1G33790 & $\begin{array}{l}\text { Mannose-binding lectin superfamily } \\
\text { protein }\end{array}$ & Jacalin lectin family protein. Uncharacterized function \\
\hline Eucgr.E02849 & AT4G18930 & $\begin{array}{l}\text { RNA ligase/cyclic nucleotide } \\
\text { phosphodiesterase family protein }\end{array}$ & $\begin{array}{l}\text { RNA ligase/cyclic nucleotide phosphodiesterase family } \\
\text { protein }\end{array}$ \\
\hline Eucgr.B00742 & AT1G68390 & Core-2/I-branching beta-1 & $\begin{array}{l}\text { Core-2/I-branching beta-1,6-N-acetylglucosaminyltransfer } \\
\text { ase family protein }\end{array}$ \\
\hline Eucgr.E00385 & AT2G21840 & $\begin{array}{l}\text { Cysteine/Histidine-rich } \mathrm{C} 1 \text { domain } \\
\text { family protein }\end{array}$ & $\begin{array}{l}\text { Zinc ion binding and involved in intracellular signaling } \\
\text { pathway }\end{array}$ \\
\hline Eucgr.B03175 & - & Remorin family protein & - \\
\hline Eucgr.A02070 & AT3G04070 & NAC domain containing protein 47 & $\begin{array}{l}\text { multicellular organismal development, regulation of } \\
\text { transcription, transcription factor (NAC047) }\end{array}$ \\
\hline Eucgr.L02157 & AT5G45470 & $\begin{array}{l}\text { Protein of unknown function } \\
\text { (DUF594) }\end{array}$ & Unknown function (DUF594). \\
\hline Eucgr.A02133 & AT3G03750 & SET domain protein 20 & $\begin{array}{l}\text { zinc ion binding, histone-lysine N-methyltransferase } \\
\text { activity and chromatin modification }\end{array}$ \\
\hline Eucgr.K01344 & AT2G32270 & zinc transporter 1 precursor & A member of Zrt- and Irt-related protein (ZIP) family. \\
\hline
\end{tabular}


transcriptTranscript is induced in response to zinc deficiency in the root. Also, response to iron deficiency.

\begin{tabular}{lll} 
Eucgr.A00929 AT3G21760 & $\begin{array}{l}\text { UDP-Glycosyltransferase } \\
\text { protein }\end{array}$ & $\begin{array}{l}\text { superfamily inhibitor of cell expansion in vivo to form a bioactive } \\
\text { glucoside }\end{array}$ \\
\hline
\end{tabular}

Encodes a protein with high affinity, hexose-specific/H+

Eucgr.H03539 AT5G26340 Major facilitator superfamily protein symporter activity. The activity of the transporter appears to be negatively regulated by phosphorylationL5

GA-responsive GAST1 protein homolog regulated by BR

Eucgr.F00591 AT1G75750 GAST1 protein homolog 1 and GA antagonistically. Possibly involved in cell elongation based on expression data

\begin{tabular}{llll}
\hline Eucgr.L01281 & AT1G60420 & DC1 domain-containing protein & Reduces transmission through pollen. \\
\hline Eucgr.D00326 & AT4G18210 & purine permease 10 & $\begin{array}{l}\text { May be involved in the transport of purine and purine } \\
\text { derivatives such as cytokinins, across the plasma } \\
\text { membrane. }\end{array}$ \\
\hline
\end{tabular}

Eucgr.H01528 AT1G74310 heat shock protein 101

Encodes ClpB1, which belongs to the Casein lytic proteinase/heat shock protein $100(\mathrm{Clp} / \mathrm{Hsp} 100)$ family. Involved in refolding of proteins which form aggregates under heat stress

\begin{tabular}{|c|c|c|c|}
\hline Eucgr.K01947 & AT4G20900 & $\begin{array}{l}\text { Tetratricopeptide repeat (TPR)-like } \\
\text { superfamily protein }\end{array}$ & $\begin{array}{l}\text { ms } 5 \text { mutants are male sterile, pollen tetrads undergo an } \\
\text { extra round of division after meiosis II without } \\
\text { chromosome replication, resulting in chromosome } \\
\text { abnormalities. }\end{array}$ \\
\hline Eucgr.L03554 & AT3G07880 & $\begin{array}{l}\text { Immunoglobulin E-set superfamily } \\
\text { protein }\end{array}$ & $\begin{array}{l}\text { SUPERCENTIPEDE1 (SCN1), Rho GDP-dissociation } \\
\text { inhibitor activity and involved in root epidermal cell } \\
\text { differentiation, cell tip growth. }\end{array}$ \\
\hline Eucgr.H00528 & AT3G63380 & $\begin{array}{l}\text { ATPase E1-E2 type family protein / } \\
\text { haloacid dehalogenase-like protein }\end{array}$ & $\begin{array}{l}\text { ATPase E1-E2 type family protein; calcium-transporting } \\
\text { ATPase activity; calcium ion transport located in } \\
\text { membrane }\end{array}$ \\
\hline Eucgr.L03371 & AT2G30470 & $\begin{array}{l}\text { high-level expression of } \\
\text { sugar-inducible gene } 2\end{array}$ & $\begin{array}{l}\text { Member of B3 family. Active repressor of the Spo } \\
\text { minimal promoter through the EAR motif. }\end{array}$ \\
\hline Eucgr.G02802 & AT2G16880 & $\begin{array}{l}\text { Pentatricopeptide repeat (PPR) } \\
\text { superfamily protein }\end{array}$ & Pentatricopeptide repeat (PPR) superfamily protein \\
\hline Eucgr.G01996 & AT1G65810 & $\begin{array}{l}\text { P-loop containing nucleoside } \\
\text { triphosphate hydrolases protein }\end{array}$ & $\begin{array}{l}\text { P-loop containing nucleoside triphosphate hydrolases } \\
\text { superfamily protein }\end{array}$ \\
\hline Eucgr.C00666 & AT2G26170 & cytochrome $\mathrm{P} 450$ & $\begin{array}{l}\text { Encodes a protein with similarity to thromboxane-A } \\
\text { synthase, member of the CYP711A cytochrome P450 } \\
\text { family. Expressed in vascular traces in the rosette stem } \\
\text { and axillary buds. }\end{array}$ \\
\hline Eucgr.F01779 & AT1G20640 & $\begin{array}{l}\text { Plant regulator RWP-RK family } \\
\text { protein }\end{array}$ & Plant regulator RWP-RK family protein \\
\hline Eucgr.I00729 & AT5G43120 & $\begin{array}{l}\text { ARM-repeat/Tetratricopeptide repeat } \\
\text { (TPR)-like protein }\end{array}$ & $\begin{array}{l}\text { ARM-repeat/Tetratricopeptide repeat (TPR)-like } \\
\text { protein; binding function }\end{array}$ \\
\hline Eucgr.E03930 & AT3G51970 & acyl-CoA sterol acyl transferase 1 & acyltransferase activity. Localized in endomembrane \\
\hline Eucgr.G01935 & AT5G53150 & $\begin{array}{l}\text { DNA heat shock N-terminal } \\
\text { domain-containing protein }\end{array}$ & heat shock protein binding. Involved in protein folding \\
\hline Eucgr.K01950 & AT5G11720 & $\begin{array}{l}\text { Glycosyl hydrolases family } 31 \\
\text { protein }\end{array}$ & $\begin{array}{l}\text { Glycosyl hydrolases; involved in carbohydrate metabolic } \\
\text { process; located in apoplast, vacuole, plant-type cell wall }\end{array}$ \\
\hline
\end{tabular}




\begin{tabular}{llll} 
Eucgr.J00165 & AT2G40390 & - & Unknow protein \\
\hline Eucgr.J01979 & AT5G59720 & $\begin{array}{l}\text { HSP20-like chaperones superfamily } \\
\text { protein }\end{array}$ & $\begin{array}{l}\text { Encodes a low molecular weight heat shock protein that } \\
\text { contains the heat shock element in the promoter region. } \\
\text { Expression is induced in response to heat shock. }\end{array}$ \\
\hline Eucgr.K00177 & AT1G65680 & expansin B2 & member of BETA-EXPANSINS \\
\hline Eucgr.E01215 & AT5G45380 & solute:sodium symporters & Degradation of Urea (DUR3). Sodium/solute symporter \\
\hline Eucgr.E03282 & AT5G18360 & $\begin{array}{l}\text { disease resistance protein } \\
\text { (TIR-NBS-LRR class) }\end{array}$ & $\begin{array}{l}\text { Disease resistance protein family, transmembrane receptor } \\
\text { activity, nucleoside-triphosphatase activity, } \\
\text { nucleotide binding, ATP binding. Involved in signal } \\
\text { transduction, defense response, apoptosis, innate immune } \\
\text { response }\end{array}$ \\
\hline Eucgr.G00561 & AT1G77855 & - & - \\
\hline Eucgr.I01734 & AT3G27550 & $\begin{array}{l}\text { RNA-binding CRS1/YhbY (CRM) } \\
\text { domain protein }\end{array}$ & RNA-binding CRS1 / YhbY (CRM) domain protein \\
\hline Eucgr.A01170 & - & - & - \\
\hline
\end{tabular}

Table 2. Spatial gene distribution between E. globulus and E. urograndis. Genes downregulated in E. globulus and independently for E. urograndis (Figure 2). Cut-off fold change: 10. We also indicate the Arabidopsis homolog, description and function using TAIR database.

\subsection{Expression profile of selected genes focused in cold and economical traits for Eucalyptus}

RNA-seq provided a broad gene expression overview and we explored the data from Figure 1 to identify the most differentially expressed genes in Eucalyptus species. In addition, we selected based on literature cell wall related genes. Kinases are intrinsically related to cold response and affects the cell wall dynamics. Several proteins area activated by phosphorylation and kinases are the first step as a response to cold stress and they allow for the fluidity of the membrane to keep form, avoiding freezing and, consequently, cell damage ${ }^{[10]}$. Here, we selected, using GO annotation, all the kinases in both species and summarized and annotated them in Figure 3. A total of 62 kinases were identified and several showed high distinct patterns of expression. Two kinases are associated with cell wall and might be involved in response to cold stress: Eucgr.C03129 and Eucgr.F04287. Eucgr.C03129 is downregulated in E. globulus. Eucgr.F04287 is differently upregulated in E. globulus.

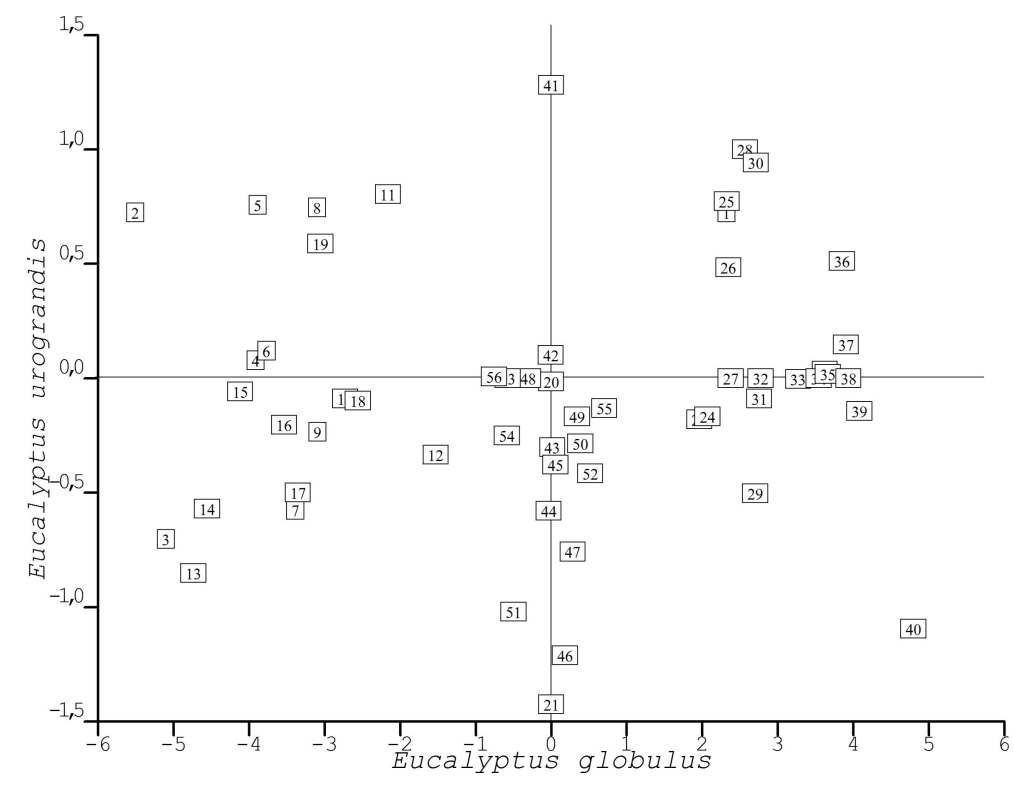

Figure 3; Spatial distribution of kinases between E. globulus and E. urograndis.

The $\mathrm{x}$ and $\mathrm{y}$ axes indicate fold change in cold response. List of genes: (1)Eucgr.A00282; (2)Eucgr.A01154; 
(3)Eucgr.A01155; (4)Eucgr.A01482； (5)Eucgr.A01762； (6)Eucgr.A01765; (7)Eucgr.B00290; (8)Eucgr.B00556; (9)Eucgr.B00766; (10)Eucgr.B00782; (11)Eucgr.B00810; (12)Eucgr.B01034; (13)Eucgr.B02528; (14)Eucgr.B03649; (15)Eucgr.C01389; (16)Eucgr.C01451; (17)Eucgr.C02358; (18)Eucgr.C03129; (19)Eucgr.C03832; (20)Eucgr.C04151; (21)Eucgr.D00446; (22)Eucgr.D00914; (23)Eucgr.D00919; (24)Eucgr.E00581; (25)Eucgr.E02450; (26)Eucgr.E03457; (27)Eucgr.E04067; (28)Eucgr.F00850; (29)Eucgr.F02419; (30)Eucgr.F02500; (31)Eucgr.F02516; (32)Eucgr.F03308; (33)Eucgr.F03765; (34)Eucgr.F03981; (35)Eucgr.F04005; (36)Eucgr.F04287; (37)Eucgr.F04434; (38)Eucgr.G00775; (39)Eucgr.G01015; (40)Eucgr.G01747; (41)Eucgr.G01897; (42)Eucgr.G02274; (43)Eucgr.H00964; (44)Eucgr.H01556; (45)Eucgr.H02636; (46)Eucgr.I01659; (47)Eucgr.J01520; (48)Eucgr.J02873; (49)Eucgr.K01759; (50)Eucgr.K03047; (51)Eucgr.L00381; (52)Eucgr.L00480; (53)Eucgr.L01599; (54)Eucgr.L02004; (55)Eucgr.L02016; (56)Eucgr.J02803; (57)Eucgr.B03317; (58)Eucgr.E00442; (59)Eucgr.E03666; (60)Eucgr.E04192; (61)Eucgr.D00784; (62)Eucgr.B01186.

Few cold responsive genes were identified and special attention was given to CBF transcription factor genes, which triggers several responses to cold ${ }^{[11]}$. There are three homolog genes for E. urograndis and E. globulus. The expression pattern is different for Eucgr.A02831 and Eucgr.A02832 agreeing with the main characteristic of E. globulus - being adapted to cold places ${ }^{[12]}$ (Figure 4). Both genes are upregulated, especially Eucgr.A02832, although this same gene is downregulated in E. grandis.

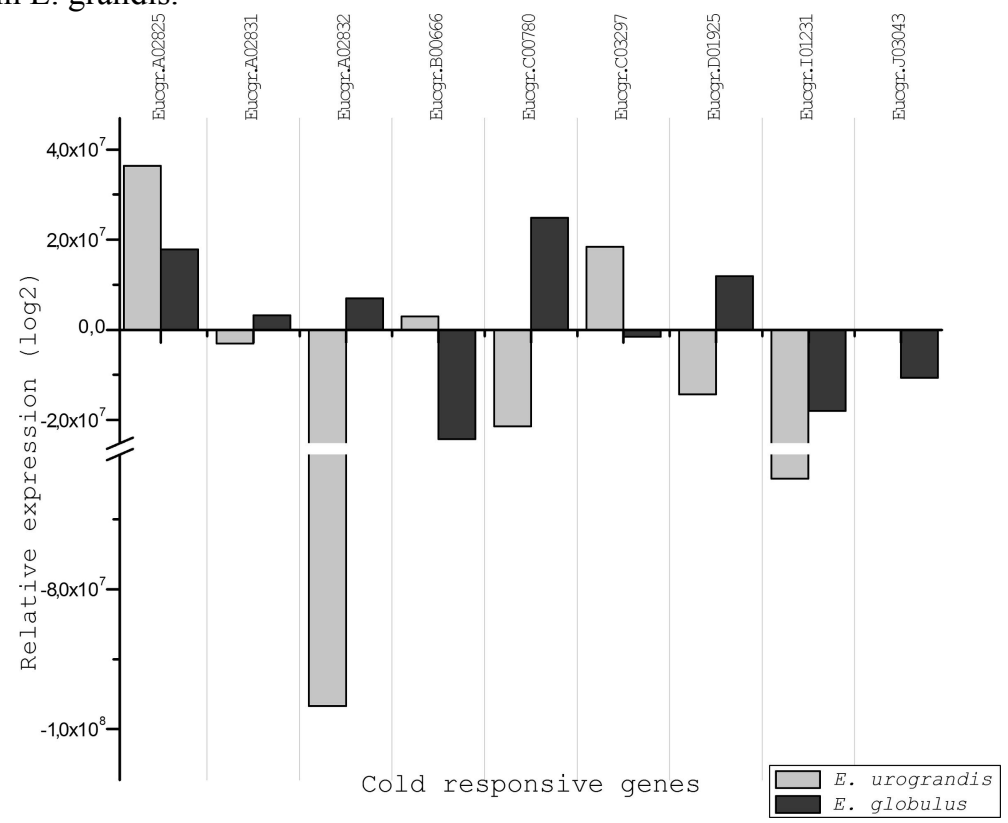

Figure 4; Expression profile of COR genes in Eucalyptus species. Annotated genes and homolog from Arabidopsis: Eucgr.A02825 (CBF1); Eucgr.A02831 (CBF1); Eucgr.A02832 (CBF1); Eucgr.B00666 (RAP2.11); Eucgr.C00780 (ERF017); Eucgr.C03297 (ERF017); Eucgr.D01925 (CBF2); Eucgr.I01231 (COR413); Eucgr.J03043 (LEA).

The lignin pathway is coordinated by several and redundant genes ${ }^{[13]}$. In general, most of the genes in this pathway are: (i) upregulated in E. urograndis and (ii) downregulated in E. globulus. The genes F5H and CAD are consistently expressed following the characteristic behavior found in these species. Most CAD genes (Eucgr.E01103, Eucgr.E01104; Eucgr.E01105, Eucgr.F01677 and Eucgr.G01350) are upregulated and can be involved in the high levels of lignin in E. urograndis due to them being on the last step of monolignol synthesis. All the F5H are upregulated in E. urograndis and three other genes are more expressed in E. globulus. This step is important in synapyl alcohol synthesis and will increase the amount of $\mathrm{S}$ lignin units creating less $\mathrm{C}-\mathrm{C}$ bonds leading to easier pulp extraction ${ }^{[13]}$. The $\mathrm{S} / \mathrm{G}$ ratio is higher in E. globulus and the transcripts Eucgr.B00716, Eucgr.C00484 and Eucgr.K02211 might be regulating S lignin in this pathway (Figure 5). 




Figure 5. Expression profile of lignin pathway genes in Eucalyptus species.

Annotated genes and homolog from Arabidopsis: Eucgr.A02185 (C3H); Eucgr.A02188 (C3H); Eucgr.G03199 (C3H); Eucgr.C00065 (C4H); Eucgr.J01844 (C4H); Eucgr.E01103 (CAD); Eucgr.E01104 (CAD); Eucgr.E01105 (CAD); Eucgr.E01115 (CAD); Eucgr.F01677 (CAD); Eucgr.F01679 (CAD); Eucgr.F01680 (CAD); Eucgr.G01350 (CAD); Eucgr.L01279 (CAD); Eucgr.C00927 (CCoAOMT); Eucgr.G01417 (CCoAOMT); Eucgr.I01134 (CCoAOMT); Eucgr.A01397 (COMT); Eucgr.E03148 (COMT); Eucgr.F02623 (COMT); Eucgr.H03922 (COMT); Eucgr.K00957 (COMT); Eucgr.B00712 (F5H); Eucgr.B00716 (F5H); Eucgr.C00484 (F5H); Eucgr.I02371 (F5H); Eucgr.J02393 (F5H); Eucgr.K02211 (F5H); Eucgr.K02212 (F5H); Eucgr.C04087 (4CL).

Transcription factors reported in the literature to be related with wood formation were also selected for analysis ${ }^{[14]}$ (Figure 6). In E. globulus, Eucgr.D00594 a homolog of SND2 was downregulated within auxin responsive genes. Eucgr.G00390 and Eucgr.G00392 are upregulated in E. globulus. The expression level of Eucgr.A01767 (MYB15) in E. urograndis was 8-fold as in E. globulus, possibly being be related to ICE1, which controls MYB15 negatively and promotes $\mathrm{CBF} 3$ expression $^{[15]}$. In the same way, the cold treatment is stressful for E. urograndis and might regulate this differential expression of Eucgr.A01767. Eucgr.C03153 (MYB32) is upregulated in E. urograndis and is highly induced by MYB46. On the other hand, in E. globulus, Eucgr.C03153 is downregulated and may be related to lower lignin deposition, contrasting to E. urograndis phenotype. Eucgr.C03151 and Eucgr.J02817 are closely related to MYB4 from Arabidopsis, which inhibited the expression of hydroxycinnamate ester biosynthesis and affected UV-B tolerance (increased in myb4 by the absence of inhibition) ${ }^{[16]}$. $\mathrm{C} 4 \mathrm{H}$ (cinnamate 4-hydroxylase) from lignin pathway is a second key enzyme coordinating the cinnamic acid production after $\mathrm{PAL}^{[13]}$. Thus, Eucgr.C03151 is upregulated in E. urograndis and Eucgr.J02817 is downregulated in both species. 


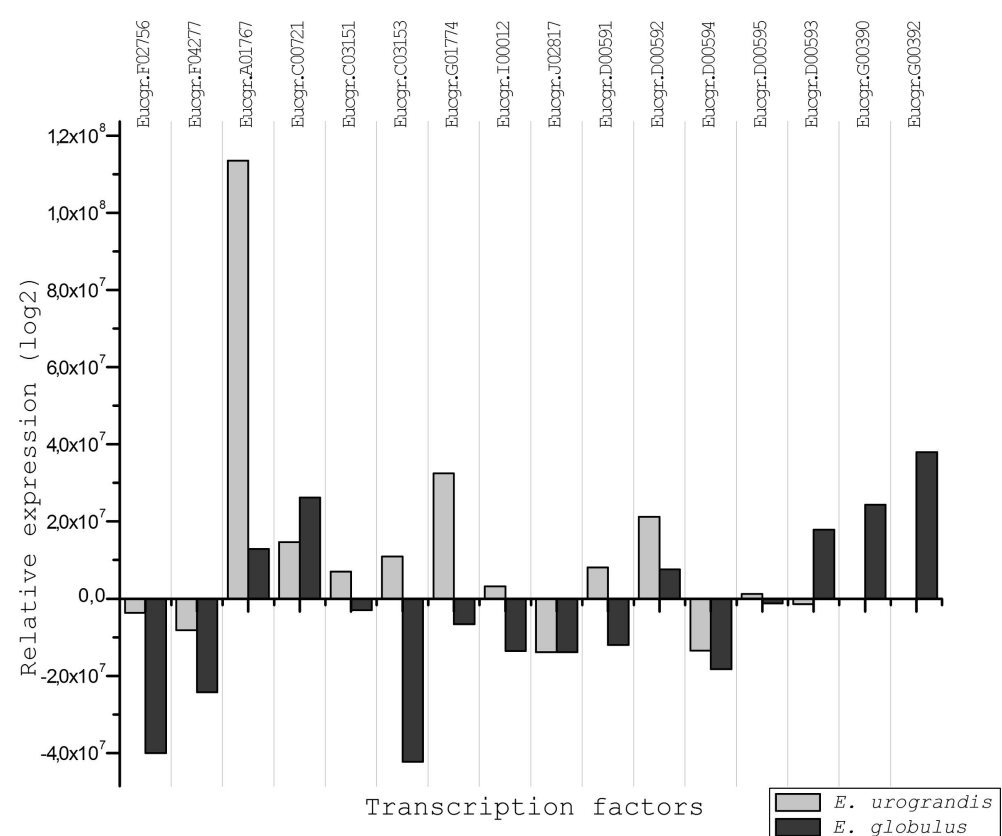

Figure 6. Expression profile of transcription factors related to lignin regulation in Eucalyptus species.

Annotated genes and homolog from Arabidopsis: Eucgr.F02756 (MYB52); Eucgr.F04277 (MYB52); Eucgr.A01767 (MYB15); Eucgr.C00721 (MYB7); Eucgr.C03151 (MYB4); Eucgr.C03153 (MYB32); Eucgr.G01774 (MYB4); Eucgr.I00012 (MYB7); Eucgr.J02817 (MYB4); Eucgr.D00591 (SND2); Eucgr.D00592 (SND2); Eucgr.D00594 (SND2); Eucgr.D00595 (SND2); Eucgr.D00593 (SND2); Eucgr.G00390 (ERF-13); Eucgr.G00392 (ERF-13).

Cellulose is the main economical product from Eucalyptus and it is used for paper production and synthesized by CELLULOSE SYNTHASE (CesA $)^{[17]}$. Cellulose biosynthesis is intrinsically coordinated by NAC and MYB transcription factors which are responsible for primary and secondary cell wall deposition ${ }^{[18]}$. Only in E. globulus homologs CesA genes were upregulated, corresponding to CesA3, CesA5, CesA6 and possibly relating with better development of E. globulus under low temperature ${ }^{[12,17]}$ (Figure 7).

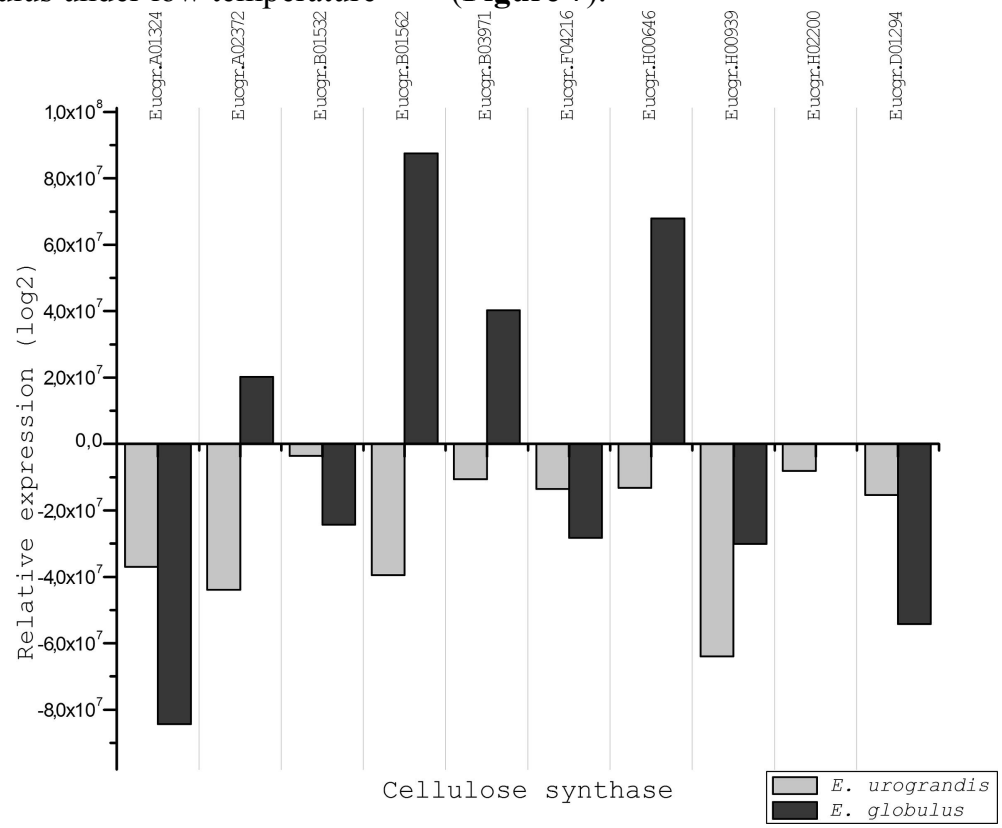

Figure 7; Expression profile of cellulose synthase genes in Eucalyptus species.

Annotated genes and homolog from Arabidopsis: Eucgr.A01324 (CesA4); Eucgr.A02372 (CesA3); Eucgr.B01532 (CesA6); Eucgr.B01562 (CesA6); Eucgr.B03971 (CesA5); Eucgr.F04216 (CesA6); Eucgr.H00646 (CesA6); Eucgr.H00939 (CesA1); Eucgr.H02200 (CesA9); Eucgr.D01294 (XTH8).

Expansin regulates relaxation of the cell wall allowing cell expansion and growth. In both Eucalyptus, 
Eucgr.A00988 and Eucgr.F03723 are downregulated in E. urograndis and upregulated in E. globulus. On the other hand, Eucgr.E01615 is upregulated in E. globulus and all the other transcripts keep the same pattern in both species (Figure 8).

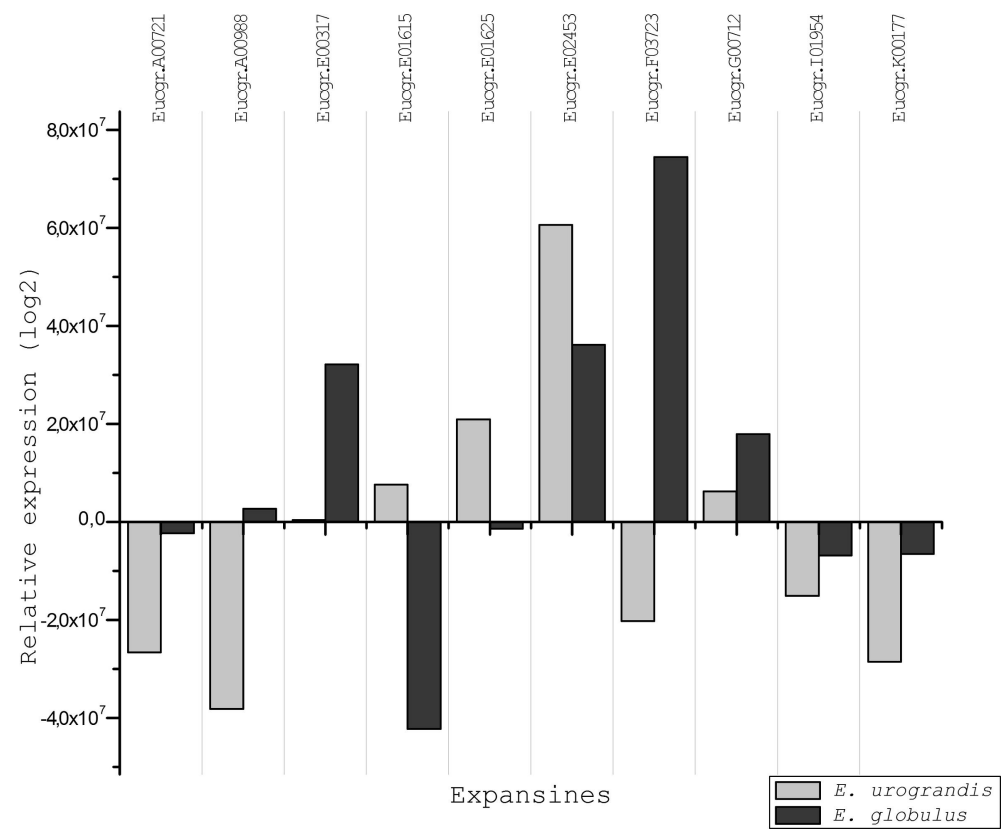

Figure 8; Expression profile of expansin genes in Eucalyptus species.

Annotated genes and homolog from Arabidopsis: Eucgr.A00721 (EXPA10); Eucgr.A00988 (EXPA17); Eucgr.E00317 (EXLB1); Eucgr.E01615 (EXPB2); Eucgr.E01625 (EXPB2); Eucgr.E02453 (uncharacterized expansin-like); Eucgr.F03723 (EXPA11); Eucgr.G00712 (EXPA4); Eucgr.I01954 (EXPA10); Eucgr.K00177 (EXPB2).

Flavonoid related genes have the same pattern in the two species. The only differentially expressed gene is a chalcone isomerase homolog Eucgr.J01153 (Figure 9). It is a key branch-point gene of the phenylpropanoid pathway after 4CL. Chalcone isomerase catalyzes the production of flavanones, which is an important skeletal backbone for further downstream metabolites.

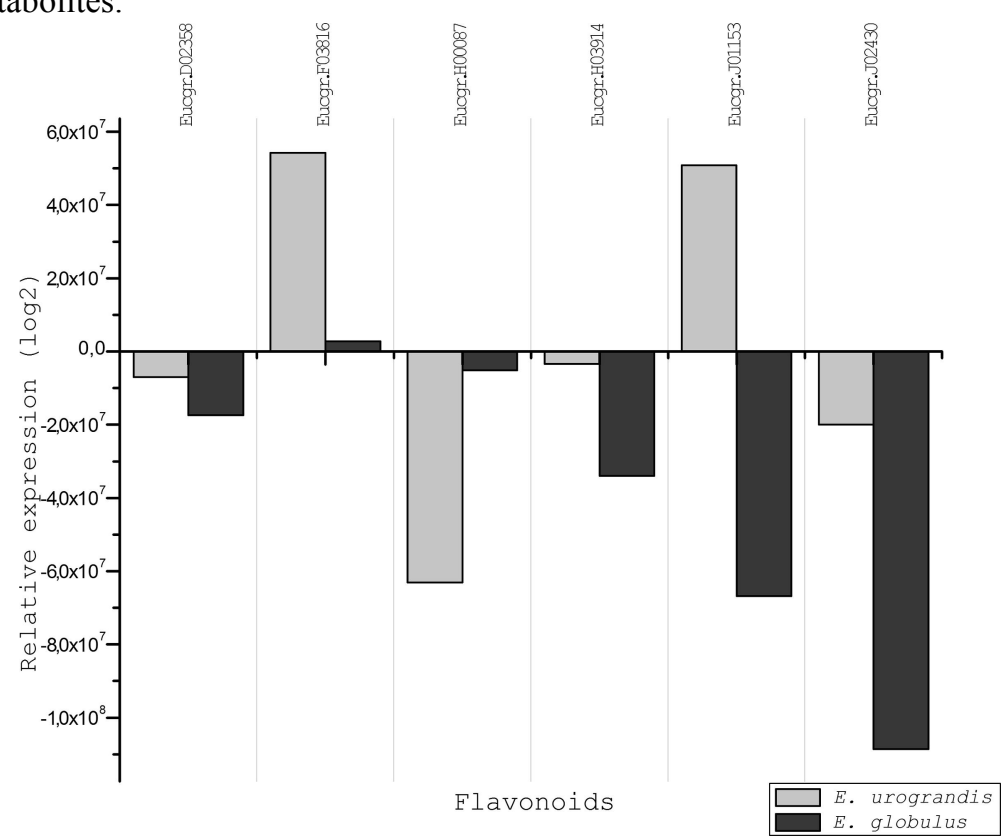

Figure 9; Expression profile of flavonoid genes in Eucalyptus species.

Annotated genes and homolog from Arabidopsis: Eucgr.D02358 (dihydroflavonol 4-reductase); Eucgr.F03816 (chalcone isomerase); Eucgr.H00087 (chalcone synthase); Eucgr.H03914 (chalcone synthase); Eucgr.J01153 (chalcone isomerase); Eucgr.J02430 (flavonone 3-hydroxylase). 
Class III peroxidases are the last step in lignin incorporation into the secondary cell wall during development ${ }^{[19]}$. This class has more than a hundred genes in Eucalyptus and correlating one of them to lignin content is a challenge. Interestingly, with the exception of only two Eucgr.F03724 (PRX64) and Eucgr.H01218 (PRX03), all the other genes are downregulated in E. globulus and upregulated in E. urograndis (Figure 10).

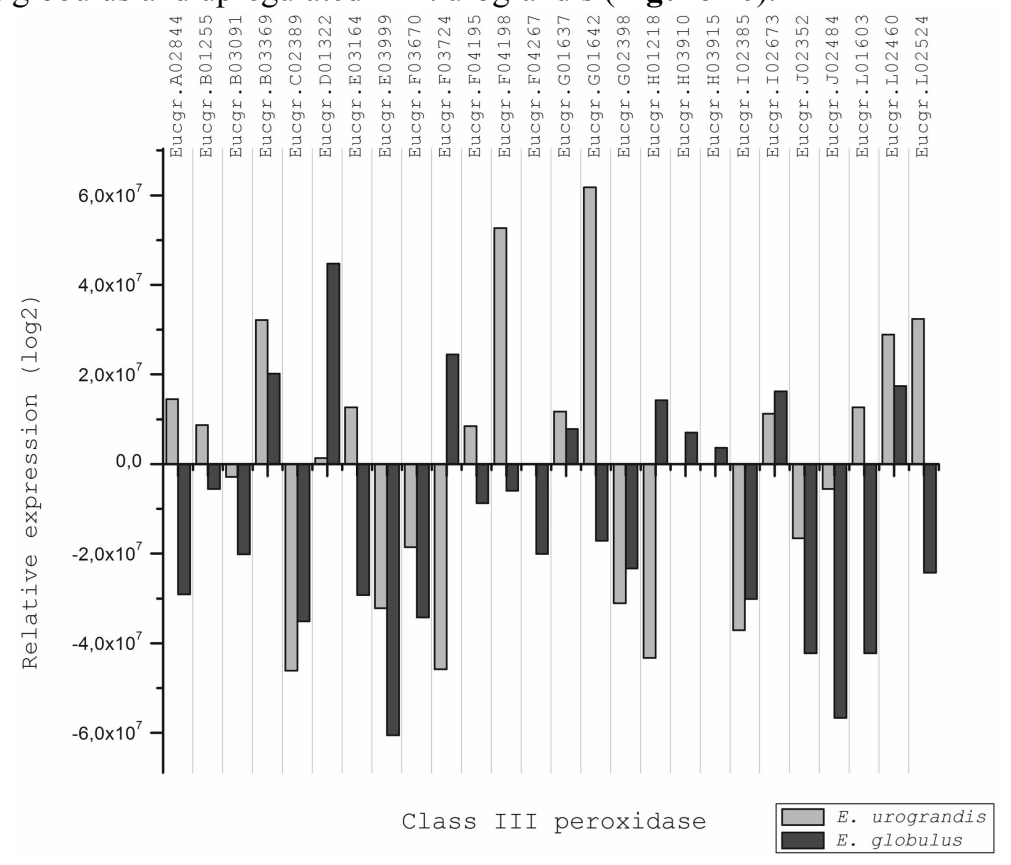

Figure 10; Expression profile of class III peroxidases genes in Eucalyptus species.

Annotated genes and homolog from Arabidopsis: Eucgr.A02844 (PRX66); Eucgr.B01255 (PRX03); Eucgr.B03091 (PRX11); Eucgr.B03369 (PRX06); Eucgr.C02389 (PRX16); Eucgr.D01322 (PRX42); Eucgr.E03164 (PRX19); Eucgr.E03999 (PRX29); Eucgr.F03670 (PRX07); Eucgr.F03724 (PRX64); Eucgr.F04195 (PRX12); Eucgr.F04198 (PRX12); Eucgr.F04267 (PRX43); Eucgr.G01637 (PRX66); Eucgr.G01642 (PRX11); Eucgr.G02398 (PRX11); Eucgr.H01218 (PRX03); Eucgr.H03910 (PRX47); Eucgr.H03915 (PRX47); Eucgr.I02385 (PRX41); Eucgr.I02673 (PRX71); Eucgr.J02352 (PRX51); Eucgr.J02484 (PRX64); Eucgr.L01603 (PRX30); Eucgr.L02460 (PRX71); Eucgr.L02524 (PRX64).

\subsection{GO annotation and physiological overview of both species under cold treatment}

The GO annotation provides a more comprehensive overview of the physiological events occurring in both species (Figure 11). The difference between E. globulus and E. urograndis is remarkable regarding ATP binding (GO:0005524) and oxidative reduction (GO:0055114). E. globulus seems to maintain the photosynthetic activity as a priority and have less oxidative stress derivate from the cold treatment. On the other hand, E. urograndis has the opposite behavior comparing both GO. The oxidative stress metabolism is a stress consequence, showing an inability during acclimatization under cold temperatures ${ }^{[20]}$. Another process pinned up in E. globulus is apoptosis (GO:0006915) which is intrinsically related to xylem differentiation and stressful condition ${ }^{[21]}$. 

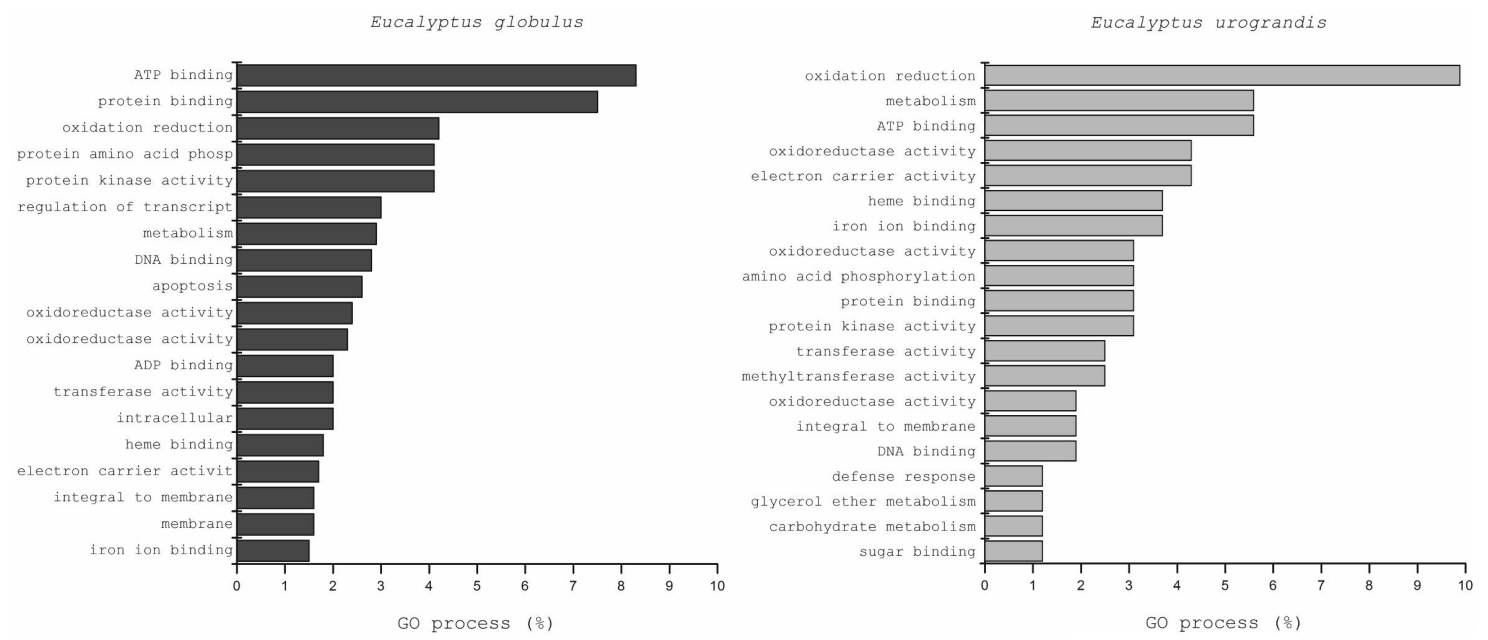

Figure 11; Most frequently observed GO processes in E. globulus and E. urograndis under cold stress.

\section{Discussion}

The RNA-seq approach reveals a large number of stress-regulated genes making it difficult to evaluate the results in a complex plant physiology context. E. urograndis has approximately $45 \%$ of its genes differentially expressed and inside this proportion, $36 \%$ of these genes are shared with E. globulus and $9 \%$ are uniquely expressed. On the other hand, E. globulus has $40 \%$ of the transcripts differentially expressed and only $3.8 \%$ are uniquely expressed. Arabidopsis transcriptome can share $30 \%$ of its genes among different abiotic stresses ${ }^{[22]}$. It is perfectly clear that there is a high number of shared genes (36\%) during cold stress in both species on the Eucalyptus genus. It is important to realize that E. globulus has less expressed genes (about 5\% less than E. urograndis) and this amount is enough to produce drastic differences among the occurring GO process. Under cold stress, the oxidation process is dominant for E. urograndis, but for E. globulus it is ATP binding. Insights about different genes related to cold tolerance still need to be validated using reverse genetics for comprehensive Eucalyptus response ${ }^{[23]}$. This methodology has been used for different non-model species and can aggregate value for further researches. The results among the species using different approaches can produce non-congruent overviews.

Eucalyptus is vulnerable to freezing injury and E. globulus is more tolerant to cold conditions than E. urograndis and, at the same time, it shows better wood quality for pulp production ${ }^{[4,12]}$. Therefore, we selected important groups of genes related to wood properties. The first step for tolerance is propitiated by kinases. We identified 64 genes and two of them are related to cell-wall and previously described here, number 40 is an interesting candidate because it is highly induced in E. globulus and strongly repressed in E. urograndis. Another interesting group of kinases formed by numbers: $22,27,32,33,34,35$ and 38 . They are upregulated in E. globulus and equally regulated in E. urograndis. They may confer higher tolerance under cold treatment. Other kinases were already identified and characterized by cold induction and related to cold tolerance, indicating specificity in its activation ${ }^{[10]}$. A Camaldulin-like 38 (CML38 - AT1G76650) homolog was identified in Eucalyptus (Eucgr.B00127). It is strongly induced in E. globulus and downregulated in E. urograndis. This kinase is one of the calcium sensors for multiple cellular signals in response to environmental stresses and directly controls a downstream signaling pathways targeting different responses to stress induced responses ${ }^{[10]}$.

CBF/DREB1 controls the transcription of various genes with important functions during cold acclimation and the development of freezing tolerance. ABA hormone, salinity and drought conditions induce significantly lower levels in the expression of $\mathrm{CBF}$ genes when comparing to cold induction ${ }^{[24]}$. CBFs interact with other cold-responsive genes that have cis elements ${ }^{[24]}$. We identified the homolog Eucgr.A02825 as induced by cold stress and could be intrinsically related to cold tolerance. The homolog of MYB15 is also induced and corresponds indirectly to up regulation of ICE in E. globulus as a response to cold induction and tolerance ${ }^{[15]}$. ICE1 binds to MYC cis-element of CBF3 and activates under cold stress. The ice1 reduces chilling tolerance and cold acclimation ${ }^{[25]}$. In fact, it is already expected due to the fact that E. globulus grows/develops better than E. urograndis in cold areas. The MYB15 is also a negative regulator of 
cold tolerance, suppressing CBF activity, binding in the promoter region of these genes. We also identified some other induced transcription factors belonging to the NAC family, MYB family and COR genes and they may contribute to increase the list of genes regulated by cold stress ${ }^{[11,22]}$.

The lignin pathway genes are more prone to be downregulated in E. globulus agreeing with lower lignin content, higher levels of S lignin and consequently easier cellulose access for fermentation. Higher expression of CAD in E. urograndis could be involved in higher levels of monolignol production contributing for carbon fixation ${ }^{[13]}$. In the same way, switchgrass RNAi mutants for CAD correlates low levels of CAD with less lignin and improved sugar release. More than one CAD homologs were identified in Eucalyptus and further studies must be carried due to the fact that some of them are more related to lignin deposition and xylem differentiation as observed in Populus- a model woody plant ${ }^{[26]}$. In fact, three homologs of F5H higher expression in E. globulus and are correlated with higher levels of $\mathrm{S}$ lignin and better the delignification process ${ }^{[13]}$. Interestingly, Eucgr.J02393 homolog was characterized in Arabidopsis. A functional complementation of fah1-2 Arabidopsis mutant was performed by F5H from E. globulus (Eucgr.J02393 homolog $)^{[27]}$. This gene was able to rescue syringyl units and sinapoylmalate. Thus, the high levels of syringyl lignin fit with E. globulus wood properties ${ }^{[4]}$. In our analysis, this gene was upregulated in E. globulus and downregulated in E. urograndis. These results strengthen this kind of genetic engineering approach for gene discovery and characterization aiming at better digestibility/saccharification.

Class III peroxidases are responsible for the radicalization and incorporation of monolignols into the lignin polymer ( $\mathrm{S}, \mathrm{G}$ and $\mathrm{H}$ units) randomly ${ }^{[19]}$. It is difficult to identify and correlate specific peroxidases with different biological processes ${ }^{[19]}$. Despite that, we observed that most PRXs are upregulated in E. urograndis and are related with oxidative stress under cold treatment - oxidative reduction is the first process on GO annotation. Plants exposed to stress are known to upregulate their overall peroxidases activity and this occurs equally for most abiotic stresses $^{[19,20]}$. Therefore, this defensive response results in stronger cell wall or ROS production ${ }^{[20]}$. In fact, it can still be related with higher levels of lignin in E. urograndis, the same does not occur for E. globulus and the only gene Eucgr.F03724, related to Arabidopsis PRX, is more expressed. The characterization of PRX for lignin deposition is still laborious.

Expansins are proteins intrinsically responsible for cell wall loosening, cell enlargement and in a variety of cell wall modifications. Four families are identified in plants: $\alpha$-expansin (EXPA), $\beta$-expansin (EXPB), expansin-like A (EXLA) and expansin-like B (EXLB). Expansin-like A and B are uncharacterized and only the gene sequence is known. Here, we identified two differentially expressed expasins, upregulated, in E. globulus which has better development and grows under cold conditions. They are potential candidates for deeper analysis. The Arabidopsis EXPA17, a homolog of Eucgr.A0098, is involved in cell modification and strongly repressed under acidic conditions. The concomitant repression of EXPA17 and other expansin-like A indicate it may limit the duration of the growth phase induced by auxin acidification. EXPA17 is regulated by auxin (IAA) and brassinosteroid in Arabidopsis ${ }^{[28]}$.

Cellulose content is an important factor for pulp production and is genetically regulated by Cellulose synthase genes (CesA). Cellulose is one of the main components in plant cell walls. It is structured into parallel unbranched ß-1, 4-glucan chains called microfibrils which consist of a well packed crystalline cellulose structure and an amorphous region. The Arabidopsis genome has 10 CesA genes and the first one characterized by the reduction of cellulose under restrictive temperatures ${ }^{[29]}$. We identified three CesA homologs upregulated in E. globulus (CesA3, 5 and 6). The cold condition does not appear to be prohibitive for CesA genes in E. globulus and can explain the expression profile observed. The Arabidopsis mutant for CesA6 has mild reduced root and elongated hypocotyl plants ${ }^{[17]}$. CesA2, CesA5 and CesA6 are partially functionally redundant and may indicate that the homologs of CesA5 and CesA6 from Eucalyptus have similar patterns of expression and function. It was observed that plants - Arabidopsis with reduced lignin content do not increase their cellulose content. However, reduced levels of CesA directly effects cell expansion and lignin synthesis, causing collapsed cell wall ${ }^{[17]}$.

Flavonoids constitute a sub-group of the phenylpropanoids that accumulate in response to variety of factors. It is a 
derivate branch from the phenylpropanoid pathway and more precisely from p-coumaroyl-CoA ${ }^{[13]}$. Recently, tricin - a member of the flavonoid family - was characterized as incorporated into the lignin polymer due to specific moieties ${ }^{[30]}$. Eucgr.J01153 is upregulated in E. urograndis and flavonoid and lignin compete for precursors. Low temperatures can induce PAL and it is know that flavonoid production depends on PAL activity, and it triggers flavonoid accumulation ${ }^{[13]}$.

\section{Conclusion}

We reported differentially expressed genes for cold tolerance using two contrasting Eucalyptus species. Our evaluation shows how they are expressed and can help to explain the physiological behavior for cold stress. We also indicate some interesting candidates for deeper analysis aiming to obtain transgenic plants. The genes somewhat focused in wood properties are able to elucidate cell wall properties - comparing with already available literature. This explanatory approach is useful to identify potential candidates for breeding.

\section{Author Contributions}

Experiments planning: JCMSMS, PA and PM; bioinformatic analysis: DF and PA; sample preparation, data organization, plot graphics: FTT, APFJ, FCS, DF, VT, NV; Manuscript writing: PA and PM.

\section{Conflict of Interest}

No conflict of interest was reported by the authors.

\section{Referance}

1. González-Garcíaa S, Moreira MT, Feijoo G. Environmental aspects of eucalyptus based ethanol production and use. Science of the Total Environment. 2012; 438:1-8.

2. Pleguezuelo CRR, Zuazo VHD, Bielders C, et al. Bioenergy farming using woody crops. A review. Agronomy for Sustainable Development. 2015; 35(1):95-119.

3. Davidson N, Reid JB. Frost as a factor influencing the growth and distribution of subalpine eucalypts. Australian Journal Botany. 1985; 33:657-667.

4. Moura JC, Bonine CA, Viana JOF, et al. Abiotic and biotic stresses and changes in the lignin content and composition in plants. Journal Integrative Plant Biology. 2010; 52(4):360-376.

5. Eldridge K, Davidson J, Hardwood C, et al. Eucalypt Domestication and Breeding. Oxford Science Publications. 1993; USA: Clarendon Press.

6. Carbonnier L, Marques C, Coutinho J, et al. The future of Eucalyptus plantations. In Borralho N, Pereira J, Marques C, et al. IUFRO on silviculture and improvement of Eucalypts: Eucalyptus in a changing world. Raiz Instituto. 2004; 29.

7. Almeida AC, Siggins A, Batista TR, et al. Mapping the effect of spatial and temporal variation in climate and soils on Eucalyptus plantation production with 3-PG, a process-based growth model. Forest Ecology and Management. 2010; 259(9):1730-1740.

8. Chinnusamy V, Zhu J, Zhu JK. Cold stress regulation of gene expression in plants. Trends in Plant Science. 2007; 12(10):444-451.

9. Wang Z, Gerstein M, Snyder M. RNA-Seq: a revolutionary tool for transcriptomics. Nature reviews Genetics. 2009; 10(1):57-63.

10. Zhan X, Zhu JK, Lang Z. Increasing Freezing Tolerance: Kinase Regulation of ICE1. Developmental Cell. 2015; 32:257-258.

11. Shi Y, Huang J, Sun T, et al. The precise regulation of different COR genes by individual CBF transcription factors in Arabidopsis thaliana. Journal Integrative Plant Biology. 2016; 59(2):118-133.

12. Tibbits WN, White TL, Gary RH, et al. Genetic variation in frost resistance of Eucalyptus globulus ssp. globulus assessed by artificial freezing in winter. Australian Journal of Botany. 2006; 54(6):521-529.

13. Boerjan W, Ralph J, Baucher M. Lignin biosynthesis. Annual Review of Plant Biology. 2003; 54:519-546.

14. Zhong R, Lee C, Zhou J, et al. A Battery of Transcription Factors Involved in the Regulation of Secondary Cell Wall Biosynthesis in Arabidopsis. Plant Cell. 2008; 20(10):2763-2782.

15. Agarwal M, Hao Y, Kapoor A, et al. A R2R3 type MYB transcription factor is involved in the cold regulation of CBF genes and in acquired freezing tolerance. Journal of Biological Chemistry. 2006; 281(49):37636-37645.

16. Jin H, Cominelli E, Bailey P, et al. Transcriptional repression by AtMYB4 controls production of UV-protecting sunscreens in Arabidopsis. Embo Journal. 2000; 19(22):6150-6161.

17. McFarlane HE, Döring A, Persson S. The Cell Biology of Cellulose Synthesis. Annual Review of Plant Biology. 2014; 65:69-94.

18. Nakano Y, Yamaguchi M, Endo H, et al. NAC-MYB-based transcriptional regulation of secondary cell wall biosynthesis in land plants. Frontiers in Plant Science. 2015; 6(288):1-18. 
19. Marjamaa K, Kukkola EM, Fagerstedt KV. The role of xylem class III peroxidases in lignification. Journal of Experimental Botany. 2009; 60(2):367-376.

20. Sewelam N, Kazan K, Schenk PM. Global Plant Stress Signaling: Reactive Oxygen Species at the Cross-Road. Frontiers in Plant Science. 2016; 7:187.

21. Schuetz M, Smith R, Ellis B. Xylem tissue specification, patterning, and differentiation mechanisms. Journal of Experimental Botany. 2013; 64(1):11-31.

22. Kreps JA, Wu Y, Chang HS, et al. Transcriptome changes for Arabidopsis in response to salt, osmotic, and cold stress. Plant Physiology. 2002; 130(4):2129-2141.

23. Navarro M, Ayax C, Martinez Y, et al. Two EguCBF1 genes overexpressed in Eucalyptus display a different impact on stress tolerance and plant development. Plant Biotechnology Journal 2011; 9(1):50-63.

24. Akhtar M, Jaiswal A, Taj G, et al. DREB1/CBF transcription factors: their structure, function and role in abiotic stress tolerance in plants. Journal of Genetics. 2012; 91(3):385-395.

25. Chinnusamy V, Ohta M, Kanrar S, et al. ICE1: a regulator of cold-induced transcriptome and freezing tolerance in Arabidopsis. Genes \& Development. 2003; 17(8):1043-1054.

26. Baucher M, Chabbert B, Pilate G, et al. Red Xylem and Higher Lignin Extractability by Down-Regulating a Cinnamyl Alcohol Dehydrogenase in Poplar. Plant Physiology. 1996; 112:1479-1490.

27. García JR, Anderson N, Le-Feuvre R, et al. Rescue of syringyl lignin and sinapate ester biosynthesis in Arabidopsis thaliana by a coniferaldehyde 5-hydroxylase from Eucalyptus globulus. Plant Cell Reports. 2014; 33(8):1263-1274

28. Lee HW, Kim J. EXPANSINA17 up-regulated by LBD18/ASL20 promotes lateral root formation during the auxin response. Plant \& Cell Physiology. 2013; 54(10):1600-1611.

29. Chen S, Ehrhardt DW, Somerville CR. Mutations of cellulose synthase (CESA1) phosphorylation sites modulate anisotropic cell expansion and bidirectional mobility of cellulose synthase. Proceedings of the National Academy of Sciences of the United States of America. 2010; 107(40):17188-17193.

30. Lan W, Lu F, Regner M, et al. Tricin, a Flavonoid Monomer in Monocot Lignification. Plant Physiology. 2015; $167: 1284-1295$ 